\title{
Synthesis and Use of C-terminally Biotinylated Peptidomimetics with High Grb2 SH2 Domain-binding Affinity
}

\section{Zhen-Dan Shi ${ }^{1}$, Benedetta Peruzzi ${ }^{2}$, Pathirage G. Dharmawardana ${ }^{2}$, Tiffany Leech ${ }^{2}$, Ettore Appella ${ }^{3}$, Karen M. Worthy ${ }^{4}$, Lakshman K. Bindu ${ }^{4}$, Robert J. Fisher ${ }^{4}$, Donald P. Bottaro ${ }^{2}$ and Terrence R. Burke, Jr. ${ }^{1}$}

${ }^{1}$ Laboratory of Medicinal Chemistry, CCR, NCI, NIH, Frederick, MD 21702 USA; ${ }^{2}$ Urologic Oncology Branch; ${ }^{3}$ Laboratory of Cell Biology, CCR, NCI, NIH, Bethesda, MD 20892 USA;

${ }^{4}$ Protein Chemistry Laboratory, SAIC-Frederick, Frederick, MD 21702 USA

\section{Introduction}

Compounds 1a and 2a bind to Grb2 SH2 domains with high affinity and exhibit interesting biochemical properties when given to whole cells in culture. In order to investigate cellular targets, biotinylated congeners were desired, however standard N-terminal biotinylation was not possible. Therefore, novel C-terminally biotinylated analogs $\mathbf{1 b}$ and $\mathbf{2 b}$ (Fig. 1) were prepared, which proved to exhibit remarkable Grb2 SH2 domain-binding affinity.
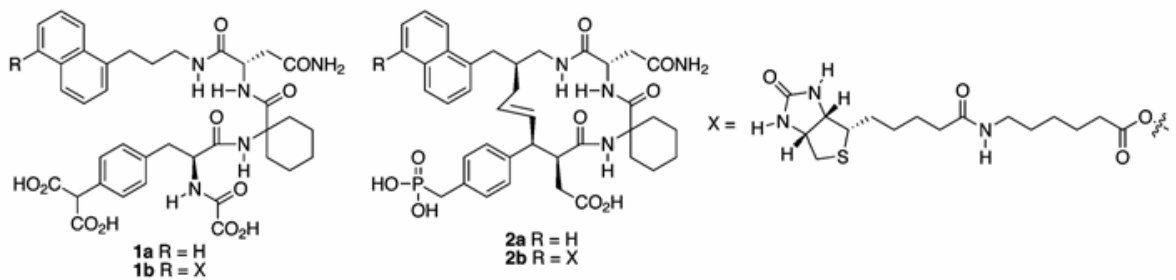

Fig. 1. Structures of analogs discussed in the text.

\section{Results and Discussion}

The biotinylated inhibitor 1b was synthesized starting from 1,5-dihydroxy naphthalene 3 by first converting to the mono-triflate ester 4 . Subsequent Heck reaction gave the expected adduct $\mathbf{5}$ as well as its reduced product $\mathbf{6}$. Reduction with $\mathrm{LiAlH}_{4}$ provided primary amine 7 (Fig. 2). This was converted to intermediate 8 then to product $\mathbf{1 b}$ following previously reported methods [1]. The synthesis of $\mathbf{2 b}$ has been in literature [1].

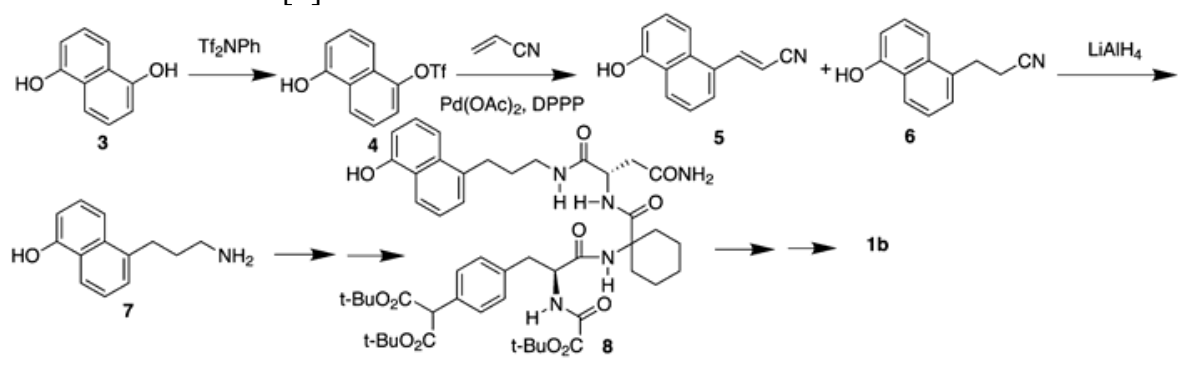

Fig. 2. Synthesis of $\mathbf{1 b}$. 
The affinities of synthetic Grb2 SH2 ligands were determined using surface plasmon resonance (SPR). SPR data $\left(k_{a}, k_{d}\right.$ and $\mathrm{K}_{\mathrm{D}}$ values) were measured for the binding of inhibitors in solution to sensor-bound Grb2 SH2 domain protein (Method a) or by binding of Grb2 $\mathrm{SH} 2$ domain protein in solution to sensor-bound inhibitor (Method b). As shown in Table 1, macrocyclic biotinylated inhibitor $\mathbf{2 b}$ had $\mathrm{K}_{\mathrm{D}}$ values in the low nanomolar range, while the $\mathrm{K}_{\mathrm{D}}$ value of the open chain biotinylated inhibitor $\mathbf{1 b}$ was an order of magnitude higher. The $k_{\mathrm{on}}$ values of both biotinylated inhibitors were similar, however the $k_{\text {off }}$ value for the macocyclic $\mathbf{2 b}$ was approximately 20 times slower than for the open-chain $\mathbf{1 b}$.

Experiments were performed to identify cellular targets: Incubation of SK-LMS1 cell lysates with $\mathbf{1 b}$ captured on streptavidin-coated beads was followed by separation of bound proteins using SDS-PAGE. Immunoblot analysis revealed that (i) Grb2 was efficiently and specifically captured from cell lysates by $\mathbf{1 b}$; (ii) Known Grb2-associated signaling proteins, e.g., Gab1, Nck1 and 2, and Sos1, as well as $\beta$ catenin were also specifically captured by $\mathbf{1 b}$; (iii) c-Met was not captured, consistent with the ability of $\mathbf{1 b}$ to antagonize Grb2/c-Met binding [2]. Work is in progress to differentiate proteins that bind directly to $\mathbf{1 b}$ from those that are brought down by secondary association with direct binding proteins.

Table 1. SPR data of the ligands binding to Grb2 SH2 domain

\begin{tabular}{lllll}
\hline Ligand & Assay Method & $\boldsymbol{k}_{\text {on }}\left(\mathbf{M}^{-\mathbf{1}} \bullet \mathbf{s e c}^{-\mathbf{1}}\right)$ & $\boldsymbol{k}_{\text {off }}\left(\mathbf{s e c}^{-\mathbf{1}}\right)$ & $\mathbf{K}_{\mathbf{D}}(\mathbf{n M})$ \\
\hline $\mathbf{2 b}$ & Biocore S51 $^{\mathrm{a}}$ & $2.24 \times 10^{6}$ & $1.78 \times 10^{-2}$ & 7.97 \\
$\mathbf{2 b}$ & Biocore S51 $^{\mathrm{b}}$ & $3.23 \times 10^{6}$ & $8.93 \times 10^{-3}$ & 2.77 \\
$\mathbf{1 b}$ & Biocore S51 $^{\mathrm{a}}$ & $1.66 \times 10^{6}$ & $2.57 \times 10^{-1}$ & 155 \\
\hline
\end{tabular}

Method a: Binding of inhibitor in solution to sensor-bound Grb2 SH2 domain protein. Method b: Binding of Grb2 SH2 domain protein in solution to sensor-bound inhibitor.

\section{Acknowledgments}

Appreciation is expressed to Drs. Christopher Lai and James Kelley of the LMC for mass spectral analysis.

\section{References}

1. Shi, Z.-D., Liu, H., Zhang, M., Roberts, L. R., Worthy, K. M., Yang, D., Fisher, R. J. and Burke, T. R. Jr. Bioorg. Med. Chem. 13, 4200-4208 (2005).

2. Dharmawardana, P., Giubellino, A., Shi, Z. -D., Burke, T. R., Jr. and Bottaro, D. (Unpublished results). 